\title{
OPEN Highly conductive electronics circuits from aerosol jet printed silver inks
}

\author{
Kacper Skarżyński ${ }^{1 凶}$, Jakub Krzemiński ${ }^{1,2}$, Małgorzata Jakubowska ${ }^{1,2}$ \& \\ Marcin Słoma $\mathbb{D}^{1 凶}$
}

Recently, low-cost electronics printed on lightweight, flexible and 3D shaped substrates are gaining importance in the markets of wearables and smart packaging. However, printed electronics do not meet the electrical performance of subtractive techniques because the resistivity of metallic printed patterns is still much higher than that of bulk material. To fulfil this need, low-resistive and easy printable inks for high resolution printed electronics techniques are needed. In this work, parameters of silver nanoparticles ink for micro-scale printed electronics technique, Aerosol Jet Printing, are being enhanced. To increase electrical conductivity and enhance printability, surfactants and dispersing agents were used to increase ultrasonic atomisation efficiency, obtain a uniform structure of printed lines, and narrow the width of printed patterns. Electrical measurements show a decrease in resistivity value in samples enhanced by cationic and non-ionic surfactants, by $95 \%$, compared to initially prepared inks. Surfactant additions to silver nanoparticles Aerosol Jet Printing ink show promising features for application in modern electronics.

Recently in the electronics industry, especially in the areas of wearables and smart packaging, the need grows to produce low-cost electronics on lightweight, flexible, and 3D substrates ${ }^{1}$. In these areas of applications, highresolution printing to ensure low dimensions of a product and high electrical conductivity for low energy consumption are crucial. To enable large scale production in such applications, printing techniques introducing high deposition speed are needed. That needs are met by direct writing techniques, allowing the contactless, additive and environmentally friendly, due to low material loss, printing of electronics. Among them, the technique combining high resolution and high printing speed with the uniform geometry and introducing the use of a wide variety of functional materials is Aerosol Jet Printing.

Aerosol Jet Printing (AJP), which schematic is shown in Fig. 1, is a printing technique that utilises a concentrated jet of atomised inks to create lines and patterns used as interconnects, passive and even active electronic components, e.g. top-gated field-effect thin-film transistor ${ }^{2}$. It is specially created for 3D substrates because created narrow aerosol jet ensures printing with nearly identical resolution in the range up to $10 \mathrm{~mm}$ from the tip of the nozzle to substrate ${ }^{3}$. This technique enables creating of interconnects of minimal width $8 \mu \mathrm{m}$ and the same order of magnitude of electrical conductivity as for bulk silver ${ }^{4}$. It also shows promise for use in various applications, such as coplanar waveguides, antennas, detection electrodes, gates for displays, and collector lines for solar cells $^{5-8}$. Due to the development of state of the art in this technique ${ }^{3,9,10}$, new process modelling approaches ${ }^{11}$ and its easy scalability, its application has started not only in research centres but also in commercial production.

Aerosol Jet Printing technique requires inks suitable to create a dense mist containing low diameter, homogenous droplets ${ }^{12}$ with high adhesion to the used substrate. Dense mist allows usage of a low total flow rate, which, with the proper sheath to mist ratio, reduces the path width dimension ${ }^{13}$. To achieve narrow lines with low resistivity, dedicated process parameters are crucial ${ }^{12}$, along with adjusting ink rheological properties.

In high-resolution applications, AJP utilises ultrasonic atomisation to create a dense aerosol mist. Schematics of the process is presented in Fig. 2. In this process, according to the cavitation-wave hypothesis by Eknadiosyants $^{14}$, droplets formation is caused by a combination of instabilities in capillary standing waves on the liquid surface and acoustic cavitation bubbles beneath the surface of a liquid.

The value of the amplitude of the ultrasonic source that transfers ultrasonic energy to the ink needs to be above the threshold to break the liquid into droplets ${ }^{15}$. Even though ultrasonic atomisation does not support as

\footnotetext{
${ }^{1}$ Micro- and Nanotechnology Division, Institute of Metrology and Biomedical Engineering, Faculty of Mechatronics, Warsaw University of Technology, 8 sw. A. Boboli st., 02-525 Warsaw, Poland. ${ }^{2}$ CEZAMAT Centre for Advanced Materials and Technologies, Poleczki St. 19, 02-822 Warsaw, Poland. ${ }^{\boxplus}$ email: kacper.skarzynski.dokt@ pw.edu.pl; marcin.sloma@pw.edu.pl
} 




Figure 1. Aerosol Jet Printing schematics (1) ink atomisation process (2) Mist flow to deposition head caused by atomisation gas flow (3) Jet concentration by sheath gas and deposition on a substrate.



Figure 2. Ultrasonic atomisation schematics (1) Ultrasonic transducer (2) Liquid ink (3) Air bubbles (4) Standing wave (5) Big droplets falling back to container due to the gravitation (6) Aerosol mist.

wide variety of material as pneumatic atomization ${ }^{16}$ (ink viscosity need to be $0-20 \mathrm{cP}$ for ultrasonic and $0-1000 \mathrm{cP}$ for pneumatic), it creates a mist containing smaller droplets. To create the smallest possible droplets, crucial parameters need to be met, such as viscosity and surface tension of the ink, also crucial for atomisation efficiency (M. Renn, "Aerosol Jet Process Development Techniques", 2010). An increase in surface tension and shear viscosity not only increase the atomisation threshold, defined as acoustic intensity $\mathrm{W} \cdot \mathrm{cm}^{-2}$ at which atomisation occurs consistently within a $10 \mathrm{~ms}$ pulse but also increase the duration time of standing wave stability, understood by an experiment on viscous fluids. Tomotika ${ }^{17}$ proposed in that research the relation of these parameters in incompressible cylindrical jets as:

$$
\tau \approx \frac{D \rho \eta}{\alpha}
$$

where:

$\tau$-duration time of jet stability.

$D$-jet diameter. 
$\rho$-density,

$\eta$-coefficient of shear viscosity.

$\sigma$-surface tension of the liquid.

Inks for aerosol jet printing with ultrasonic atomisation contains three types of ingredients: solvent, functional phase and additives. The viscosity of the ink mostly depends on used solvents ${ }^{18}$, i.e. alcohols or deionised water, as the main liquid phase.

The functional phase needs to be small scale particles to allow the creation of solvent droplets containing functional particles. Since particles of functional phase with larger diameter contained in a solvent are heavy, they are more likely to fall back to the ink container than smaller ones. The best conductive materials for printed electronics are silver, gold and copper powders, due to their good conductivity, and vast availability of nanopowders with diameters below $0,2 \mu \mathrm{m}$. Also, carbon-based micro and nanomaterials, like graphene plates and carbon nanotubes, are suitable for these applications. Their concentration in ink must be chosen precisely to achieve a trade-off between atomisation related to the formation of a standing wave in low viscosity ink and a high concentration of conductive particles in ink. Additives are used to enhance ink properties, e.g. carbon nanotubes in silver ink are used to obtain higher conductivity ${ }^{4}$. To modify the surface tension of inks, enhance the dispersion of powders and inks stability over time, surfactants additions are used ${ }^{19}$.

Surfactants are chemical compounds with the ability to change the surface tension between gas, liquid and solid phases in mixtures ${ }^{20}$. Surface tension is measured as force per unit length tending to contract the surface, preventing small fractions of liquid molecules from vaporizing ${ }^{21}$. Surfactants are amphipathic molecules that consist of a hydrophilic head group structure attached to a hydrophobic hydrocarbon or fluorocarbon chain. The hydrophilic part can be anionic, cationic, amphoteric and non-ionic. In an aqueous solution, surfactants place themselves on the solution surface because the head is attracted to liquid molecules, and the chain is attracted to gas particles, lowering the surface tension ${ }^{20}$.

Surface Active Agents properties in liquid mediums were investigated in several publications. H. Chang-Jing and K.S. Lee found out that surfactants lower the surface tension ${ }^{22}$, which decrease the atomisation threshold. It also improved its adhesion to the substrate because of better wettability, which results in a wider contact area. C.T.Kosolia found out that surfactants improve particle dispersion ${ }^{19}$, which increase the percentage of ink droplets containing functional particles in the total number of droplets. On the other hand, $\mathrm{Lu}^{23}$ found out that surfactants prevent the coalescence of water droplets in aerosol mist, decreasing the diameter of the droplets in the mist. However, no detailed research for improving silver NP ink properties for the Aerosol Jet Printing technique by surfactants has been reported.

The conductivity/resistivity of several aerosol jet printed inks was evaluated in the literature. Commonly used commercial ink UTDAgX mean conductivity was measured by A.Mahajan ${ }^{13}$, with the results of $1.12 \cdot 10^{7} \mathrm{~S} / \mathrm{m}$ and E.Cantu ${ }^{7}$, with the results of $8 \cdot 10^{6} \mathrm{~S} / \mathrm{m}$. Other most often used ink is Clariant Prelect TPS50G2, whose mean conductivity achieved by Ch. Oakley ${ }^{24}$ was $1.2 \cdot 10^{7} \mathrm{~S} / \mathrm{m}$, and M.Morales-Rodrigues ${ }^{25}$ achieve even $2.38 \cdot 10^{7} \mathrm{~S} / \mathrm{m}$. Several pieces of research modify commercial inks to improve their conductivity, and while solvent addition didn't lower the resistivity ${ }^{26}$ of ink sintered at low temperature $\left(140-160^{\circ} \mathrm{C}\right)$, SWCNT addition to ink sintered at temperature $350^{\circ} \mathrm{C}$ lower the resistivity of the commercial ink ${ }^{27}$, achieving $2.8 \cdot 10^{-8} \Omega \mathrm{m}$. A novel alternative approach of reactive silver ink solution instead of nanoparticle-based results in even higher conductivity ${ }^{4} 4.6 \cdot 10^{-7}$ $\mathrm{S} / \mathrm{m}$. However, the exact composition of commercial inks is a secret of its producer, and no detailed research for improving the conductivity of silver NP ink by surfactants have been reported.

This paper aims to develop ink with improving properties to print narrow lines with the highest conductivity. Several commercially available surfactants are evaluated, and the influence of surfactant concentration in silver NP ink on the conductivity of aerosol jest printed silver lines, sintered in a low-temperature process, is investigated. The conductivity enhancement will allow AJP to better meet the market requirements, resulting in a closer introduction of this technique for printed electronics applications.

\section{Methods}

Ink preparation. The silver nanopowder (Silvercon nAg, Fig. 3) was mixed with surfactants and dispersed in toluene. The mixture was sonicated for $30 \mathrm{~s}$ four times, using $500 \mathrm{~W}$ Sonics vibracell 534 at $40 \%$ of the power. Then it was mechanically stirred for 5 min 2 times, using Retsch RM200. After each process, the loss of toluene was refilled with alcohol-based solvent to achieve $24 \mathrm{wt}$.\% concentration of silver nanoparticles in ink. Solvents mixtures 1 and 2, used in the inks, are mixtures of organic solvents described in detail in previous work ${ }^{28,29}$.

To evaluate the influence of the surfactant concentration in ink on printing parameters, AKM-0531 (NOF Corporation) and solvent mixture 1 were used. Five different samples of basic silver ink (S0) and inks containing different concentrations of surfactant: 0.5 wt.\% (S0.5), 1 wt.\% (S1), 1.5 wt.\% (S1.5), 2 wt.\% (S2) were prepared.

In the evaluation of the influence of the type of surfactant used in ink on printing parameters, solvent mixture 2 was used. Four different samples, each containing $2 \mathrm{wt}$ \% of surfactant, of Capstone (Sigma-Aldrich non-ionic surfactant) ink (CA), Triton X100 (Digma-Aldrich non-ionic surfactant) ink (TR), AKM-0531 (NOF Corporation Cationic surfactant) ink (D2), and Span 85 (Sigma-Aldrich nonionised surfactant) ink (SP) were created.

All of the ink samples are shown in Table 1.

Deposition process. The inks were atomised and printed with an M3D Optomecs Aerosol Jet Printing system and deposited at room temperature on Kapton HN film (DuPont, surface roughness in the same order as reported in other papers ${ }^{30-32}$ ). Air was used as the carrier gas. Figure 4 contains the standard pattern design for the four-point resistivity test. For each circuit, 4 layers were deposited with sheath gas flow $40 \pm 5 \mathrm{sccm}$, carrier gas flow $26 \pm 4 \mathrm{sc} \mathrm{cm}$, and printing speed $1.5 \mathrm{~mm} / \mathrm{min}$. All groups of samples were printed separately and sintered in the low-temperature photonic-sintering process $^{33}$ by a $500 \mathrm{~W}$ halogen lamp at a $2 \mathrm{~cm}$ distance for $1 \mathrm{~min}^{34}$. 


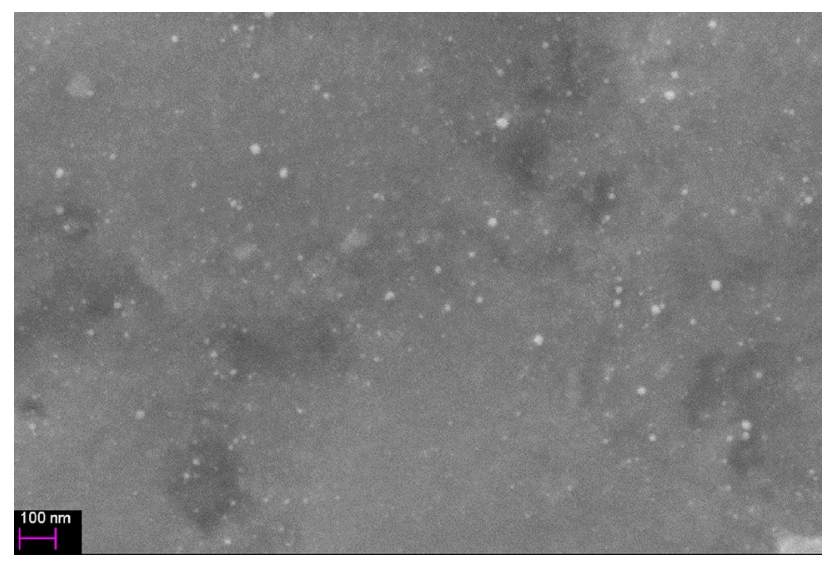

Figure 3. SEM Image of PAL silver nanoparticles, provided by the supplier. Observation has been made on scanning electron microscope LEO 1530.

\begin{tabular}{|l|l|l|l|}
\hline Ink name & Solvent mixture type & Surfactant type & Surfactant concentration (\%) \\
\hline S0 & 1 & AKM-0531 & 0 \\
\hline S0.5 & 1 & AKM-0531 & 0.5 \\
\hline S1 & 1 & AKM-0531 & 1 \\
\hline S1.5 & 1 & AKM-0531 & 1.5 \\
\hline S2 & 1 & AKM-0531 & 2 \\
\hline CA & 2 & Capstone FS-3100 & 2 \\
\hline TR & 2 & Triton X100 & 2 \\
\hline D2 & 2 & AKM-0531 & 2 \\
\hline SP & 2 & Span 85 & 2 \\
\hline
\end{tabular}

Table 1. Ink Samples for surfactant concentration test (S0-S2) and surfactant type test (CA, TR, D2, SP).



Figure 4. Pattern for four-point resistivity test (a) pattern design; (b) patterns printed on Kapton film and IR sintered.

Measurements. A four-point method for resistance measurements was used with Ketley 2001 multimeter. This method is used because of its precision in measurements of low resistances. The electrical resistivity was calculated from the equation:

$$
\rho=\frac{R \cdot S}{L}
$$

where:

$\rho$-electrical resistivity.

$S$-cross-sectional area of the printed line. 


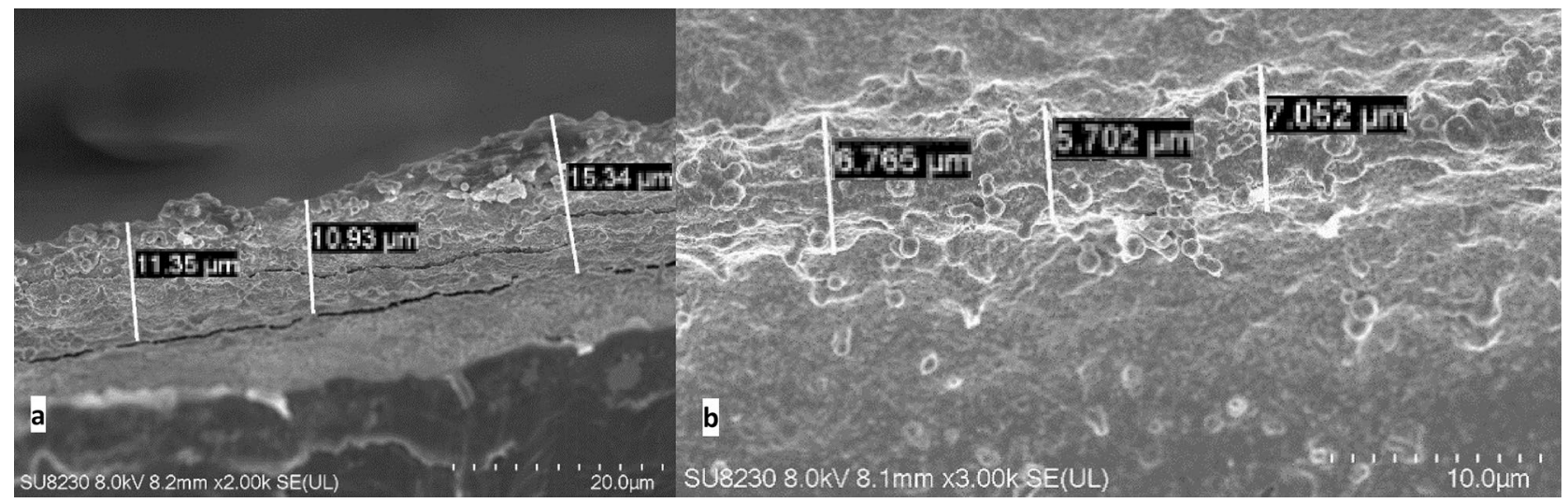

Figure 5. Measurements of the pattern, printed using S2 Ink, using Scanning Electron Microscopy images (Auriga 60) (a) the line width; (b) the line height.

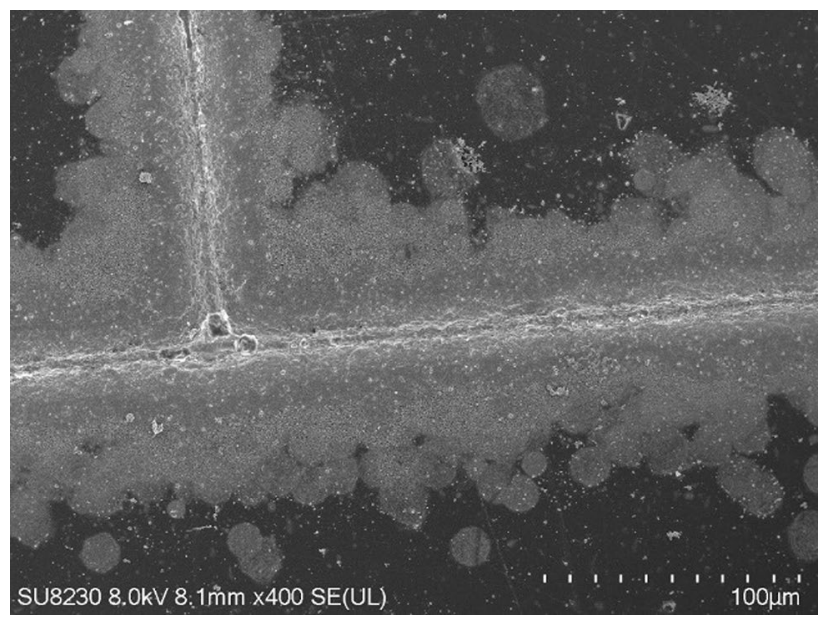

Figure 6. Overspray, characteristic Aerosol Jet Printing patterns element, emanating from the ink stream and expended by Saffman force ${ }^{36}$, visible for sample S2, in Scanning Electron Microscopy image (Auriga 60).

$L$-line length.

$R$-measured resistance.

The cross-sectional area used for the calculation of the electrical resistivity was used from the SEM observations on Auriga 60, calculated by multiplying line width and height with additional profile factor, also calculated from the SEM observations. Measurements of droplet diameter and minimum carrier gas flow that can carry mist droplets thru the aerosol system were made by deposition of aerosol droplets on filter paper with the minimum gas flow. Gas flow was measured by Bronkhorst EL_FLOW F-201CV-100-AGD-33-V flow controller, the presence of droplets marks was investigated by a 50 -fold optical microscope, and their diameter was measured by VHX-900F Keyence microscope.

\section{Results and discussion}

Structure of printed lines. The morphology of the printed lines was investigated using Scanning Electron Microscope. In Fig. 5, the observation sample results for pattern printed using S2 test ink are presented. The measurements of mean line width differ from results acquire with an optical microscope because of the overspray shown in Fig. 6. The mean line width is $7.2 \mu \mathrm{m}$, and the line height is $9.8 \mu \mathrm{m}$ (standard deviation $0.87 \mu \mathrm{m}$ ), and those parameters were used to calculate the resistivity of patterns.

The effective cross-section area used in the calculations of the resistivity is estimated from the profile of the printed path, observed with the SEM microscope in the side view of the printed samples (Fig. 7). With the use of image editing software, we have calculated that the effective conductive area of the path is only $57 \%$ of the profile calculated by the multiplication of width and height of the layer, what is comparable to results accessed by other researchers ${ }^{4}$.

Influence of Surfactants on the resistance of printed lines. Table 2 shows the mean resistance and cross-sectional area of all sample groups. Figure 7 shows the computed mean resistivity. 


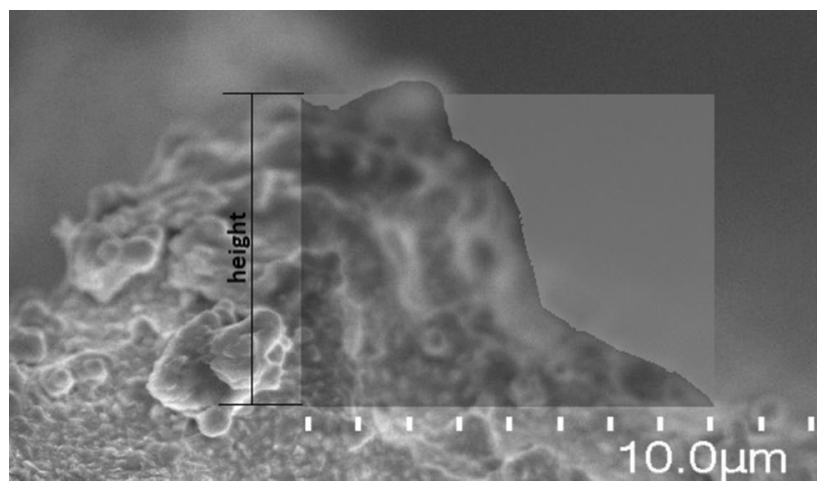

Figure 7. SEM micrograph of the cross-section profile obtained in the side view of the printed sample, with the additional image processing analysis used for the calculation of the effective profile of the printed path.

\begin{tabular}{|l|l|l|l|l|}
\hline Ink name & Mean resistance $(\boldsymbol{\Omega})$ & Mean width $(\boldsymbol{\mu m})$ & Mean droplet mark diameter $(\boldsymbol{\mu m})$ & Minimum carrier gas flow $(\mathbf{s c c m})$ \\
\hline S0 & 78.75 & 39.5 & 17.8 & 16 \\
\hline S0.5 & 51.4 & 30 & 19.2 & 16 \\
\hline S1 & 48.25 & 20.8 & 18.4 & 16 \\
\hline S1.5 & 63.9 & 17.5 & 17.5 & 16 \\
\hline S2 & 16.6 & 19.5 & 10.0 & 16 \\
\hline D2 & 18.3 & 35.4 & 8.0 & 20 \\
\hline SP & 19.3 & 49.2 & 12.0 & 20 \\
\hline TR & 50.1 & 50.2 & 50 & $0^{*}$ \\
\hline CA & $>1$ M & - & 16.5 & 20 \\
\hline
\end{tabular}

Table 2. Mean value of resistance and width acquire with an optical microscope of 8 printed $15 \mathrm{~mm}$ lines.

${ }^{\star}$ Atomisation was high enough to place droplets on the filter without any carrier gas flow.
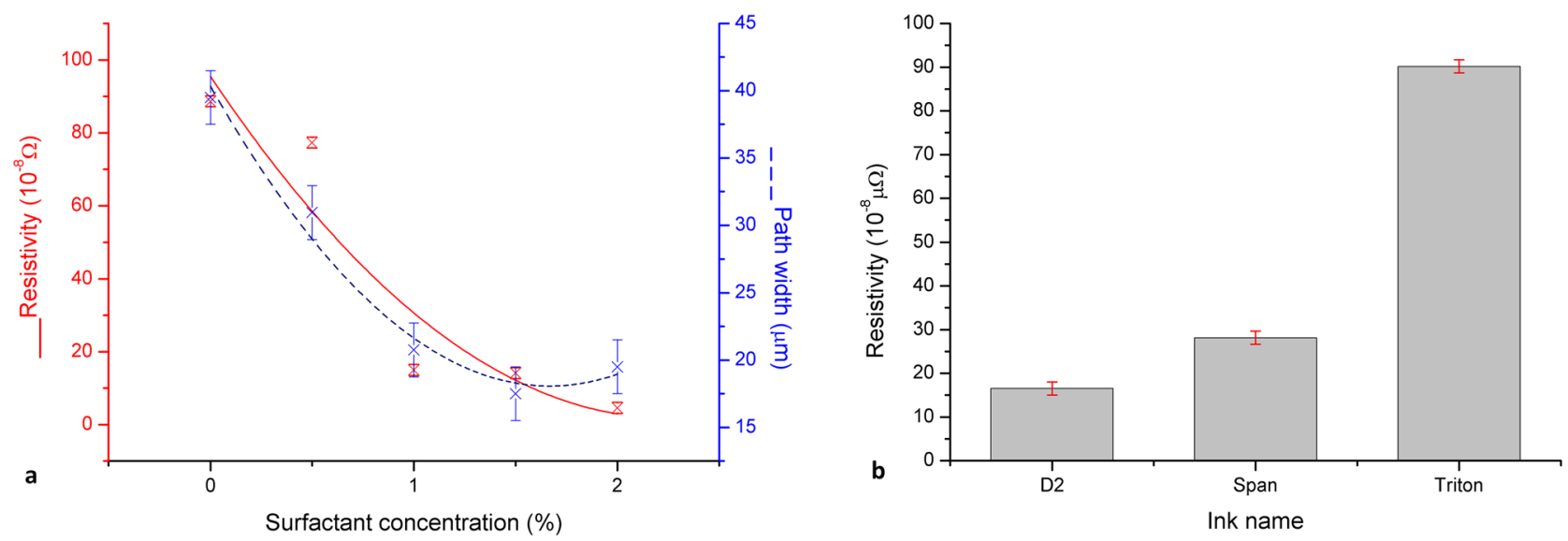

Figure 8. Charts of test results (a) computed mean resistivity and the path width for surfactant concentration test-Inks containing AKM-0531 as the surfactant in concentrations $0.5 \mathrm{wt} . \%$ (S0.5), 1 wt.\% (S1), 1.5 wt.\% (S1.5) and 2 wt.\% (S2); (b) computed mean resistivity for surfactant type test-nks containing 2 wt.\% of Capstone (CA) — not conductive, Triton X100 (TR), AKM-0531 (D2), and Span 85 (SP).

Surfactant concentration test shows that the addition of 2 wt.\% AKM-0531 surfactant lowers the electrical resistivity by $95 \%$. The addition of $0.5,1$ and $1.5 \mathrm{wt} . \%$ also improve resistivity, respectively. Figure 8 Shows that between the values 1.5 and 2 wt. $\%$ of surfactant addition, the measured mean width of the path increases. Because of this fact and the aim of this work to achieve narrow lines, higher surfactant concentrations will not be tested.

Surfactant type test shows that the electrical resistivity of printed lines containing AKM-0531 was 4 times lower than ones containing Triton X100. Lines printed using Span 85 has a higher resistivity than AKM-0531 lines but lower than Triton X100 ones, and Capstone lines were not conductive. 


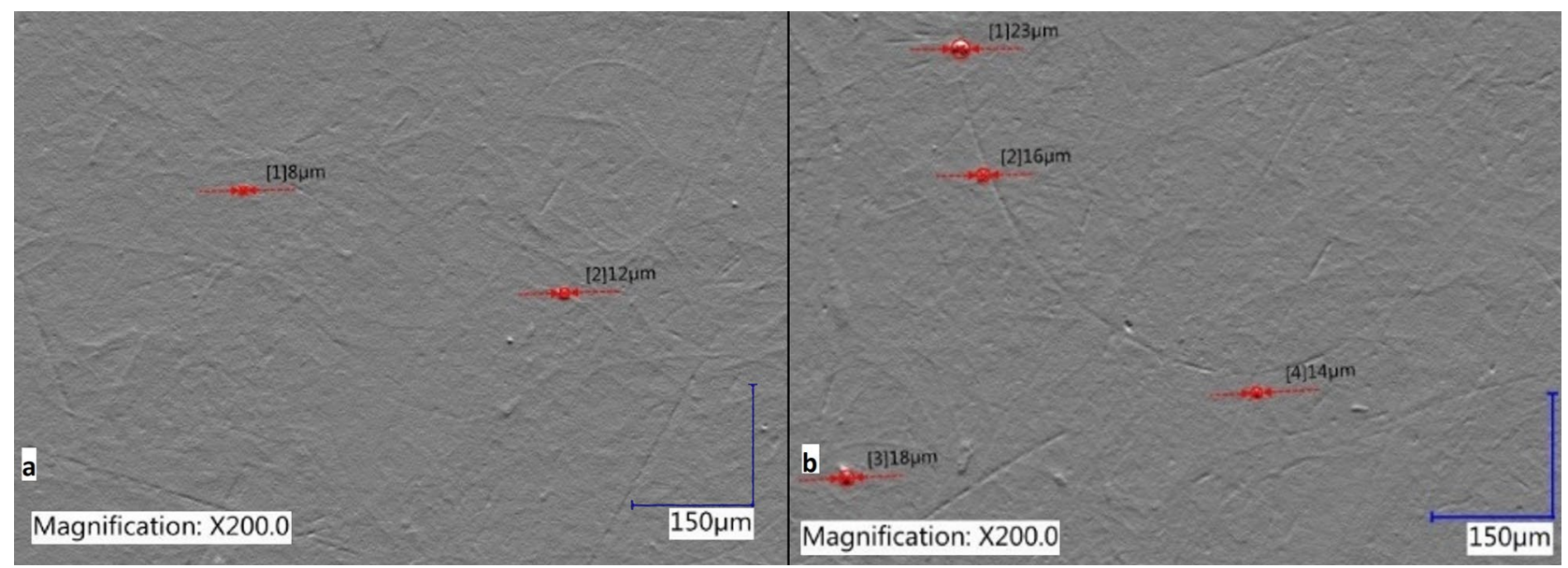

Figure 9. Example of measurements of droplet marks diameters for (a) S0 Sample; (b) S2 Sample.

The result values calculated for D2 sample ink-resistivity $4.5 \cdot 10^{-8} \Omega \mathrm{m}$ or conductivity $2.22 \cdot 10^{7} \mathrm{~S} / \mathrm{m}$-are comparable to the best results for commercial inks, and five times better than achieved in research on modification of commercial ink in which sintering was conducted in comparable temperatures $\left(2.3 \cdot 10^{-7} \Omega \mathrm{m}\right)^{26}$. A higher value of conductivity was achieved in research where authors used an alternative approach of reactive silver ink solution instead of nanoparticle-based $\left(4.6 \cdot 10^{-7} \mathrm{~S} / \mathrm{m}\right)^{27}$ and lower value of resistivity in research where the authors add carbon nanotubes to commercial ink and sinter them at temperature $350{ }^{\circ} \mathrm{C}\left(2.8 \cdot 10^{-8} \Omega \mathrm{m}\right)^{4}$.

Structural homogeneity of the printed lines, which can be enhanced with smaller droplets and lower gas flow, is the main factor that affects the electrical conductivity ${ }^{4}$. Droplet sizes and minimum gas flows were measured to investigate the results of conductivity measurements.

Table 2 shows also measured droplet marks diameters (Fig. 9) and minimum carrier gas flow values. Mean droplet marks diameter decrease with the higher increase of the surfactant concentration. The lowest diameter was measured for the droplet marks from "Triton X100" ink.

Droplet mark diameters can be used for the comparison of different samples but not to directly determine the diameter of droplets. To investigate differences between marks on a paper filter and Kapton film, lines were printed on both of them using the same inks. This resulted in an $18 \%$ wider mean line width on the paper filter. We found that a decrease in the resistivity of printed lines is directly related to an increase in the surfactant concentration in used inks, thus decreasing droplets diameter. In Fig. 5, the silver pattern is porous and, therefore, can be considered air dispersed in silver. In that silver-air system, smaller droplets decrease the air amount. According to the resistivity-mixture rule, presented by Kasap ${ }^{35}$, if the dispersed phase is at least ten times more resistive with respect to the matrix, then:

$$
\rho_{e f f}=\rho_{c} \frac{\left(1+\frac{1}{2} \chi_{d}\right)}{\left(1-\chi_{d}\right)}
$$

where:

$\rho_{\text {eff }}$-the resistivity of the entire material,

$\rho_{c}$-the resistivity of the continuous phase.

$\chi_{d}$-is the volume fraction of the dispersed phase.

For the S2 sample, which has the lowest resistivity, $\chi_{d}$ - the volume of air in the porous pattern is calculated, taking into consideration $\rho_{\text {eff }}$ as the resistivity of the printed pattern, and $\rho_{c}$ as the resistivity of the bulk silver:

$$
\chi_{d}=\frac{\frac{\rho_{e f f}}{\rho_{c}}-1}{\frac{\rho_{e f f}}{\rho_{c}}+\frac{1}{2}}=\frac{\frac{4,5 \cdot 10^{-8} \Omega m}{1,59 \cdot 10^{-8} \Omega m}-1}{\frac{4,5 \cdot 10^{-8} \Omega m}{1,59 \cdot 10^{-8} \Omega m}+\frac{1}{2}}=55 \%
$$

Triton ink results differ from the rest. That can be explained by the dependence of nozzle exit and optimum droplet diameters ${ }^{36}$. If droplets diameter is too low, line width spread significantly due to low Saffman force, which collimates bigger particles. Higher spread results in wider overspray and lesser homogeneity.

\section{Conclusions}

In this paper, we present the preparation and printing of silver NP Inks with the addition of several types of surfactant (Triton X100, Capstone, Span 85, AKM-0531) with different concentrations-0, 0.5, 1, 1.5, 2 wt.\%. Printed patterns were tested to define which surfactant concentration and type result in the lowest values of electrical resistivity. The best values of resistivity $4.5 \cdot 10^{-8} \Omega \mathrm{m}$ were obtained for the S2 sample (ink containing the addition of 2 wt.\% AKM-0531 and solvent mixture 1). The SEM observations allowed the measurements of the cross-section areas and revealed the presence of wide overspray, which occur due to smaller particle diameter caused by surfactants addition. However, it increases the density of ultrasonic mist and decreases the diameter of droplets, which results in more homogenous patterns. We prove that cationic and non-ionic surfactants, even in 
small concentrations, decrease the resistivity of printed patterns. This presents surfactants as promising additions to silver NP Aerosol Jet Printing ink for high conductive, flexible electronics.

\section{Data availability}

The datasets generated during and/or analysed during the current study are available from the corresponding author on reasonable request.

Received: 15 April 2021; Accepted: 24 August 2021

Published online: 13 September 2021

\section{References}

1. Navratil, J., Hamacek, A., Reboun, J. \& Soukup, R. Perspective methods of creating conductive paths by Aerosol Jet Printing technology. Proc. Int. Spring Seminar Electron. Technol. 38, 36-39 (2015).

2. Jones, C. S., Lu, X., Renn, M., Stroder, M. \& Shih, W. S. Aerosol-jet-printed, high-speed, flexible thin-film transistor made using single-walled carbon nanotube solution. Microelectron. Eng. 87, 434-437 (2010).

3. Feng, J. Q. Mist flow visualization for round jets in Aerosol Jet ${ }^{\star}$ printing. Aerosol Sci. Technol. 53, 45-52 (2019).

4. Zhao, D. et al. Conductivity enhancement of aerosol-jet printed electronics by using silver nanoparticles ink with carbon nanotubes. Microelectron. Eng. 96, 71-75 (2012).

5. Stoukatch, S. et al. Evaluation of Aerosol Jet Printing (AJP) technology for electronic packaging and interconnect technique. in 2012 4th Electronic System-Integration Technology Conference 1-5 (2012). https://doi.org/10.1109/ESTC.2012.6542067.

6. Cai, F. et al. Aerosol jet printing for 3-D multilayer passive microwave circuitry. in 2014 44th European Microwave Conference 512-515 (2014). https://doi.org/10.1109/EuMC.2014.6986483.

7. Cantù, E. et al. Aerosol jet printed 3D electrochemical sensors for protein detection. Sensors Switzerland 18, 3719 (2018).

8. King, B. H. \& Renn, M. J. Aerosol Jet ${ }^{\circledR}$ Direct Write Printing for Mil-Aero Electronic Applications. Optomec Inc. https://www.optom ec.com/wp-content/uploads/2014/04/Optomec_Aerosol_Jet_Direct_Write_Printing_for_Mil_Aero_Electronic_Apps.pdf (2008).

9. Salary, R. et al. Computational Fluid Dynamics Modeling and Online Monitoring of Aerosol Jet Printing Process. J. Manuf. Sci. Eng. Trans. ASME 139, https://doi.org/10.1115/1.4034591(2017).

10. Zhang, H., Choi, J. P., Moon, S. K. \& Ngo, T. H. A knowledge transfer framework to support rapid process modeling in aerosol jet printing. Adv. Eng. Inf. 48, https://doi.org/10.1016/j.aei.2021.101264(2021).

11. Salary, R. (Ross), Lombardi, J. P., Weerawarne, D. L., Rao, P. \& Poliks, M. D. A Computational Fluid Dynamics Investigation of Pneumatic Atomization, Aerosol Transport, and Deposition in Aerosol Jet Printing Process. J. Micro Nano-Manufacturing 9, https:// doi.org/10.1115/1.4049958(2021).

12. Renn, M. J., Christenson, K. K., Giroux, D. \& Blazej, D. Aerosol Jet ${ }^{\oplus}$ Printing of Conductive Epoxy for 3D Packaging. Pan Pacific Microelectron. Symp. 1-7 (2013).

13. Mahajan, A., Frisbie, C. D. \& Francis, L. F. Optimization of aerosol jet printing for high-resolution, high-aspect ratio silver lines. ACS Appl. Mater. Interfaces 5, 4856-4864 (2013).

14. Neppiras, E. A. Physical principles of ultrasonic technology. Ultrasonics 14, 138-139 (1976).

15. Rajan, R. \& Pandit, A. B. Correlations to predict droplet size in ultrasonic atomisation. Ultrasonics 39, 235-255 (2001).

16. Karl Sollner, B. The mechanism of the formation of fogs by ultrasonic waves. Trans. Faraday Soc. 32, 1532-1536 (1936).

17. Tomotika, S. Breaking up of a drop of viscous liquid immersed in another viscous fluid which is extending at aniform rate. Proc. R. Soc. Lond. A. Math. Phys. Sci. 153, 302-318 (1936).

18. Jabari, E. \& Toyserkani, E. Micro-scale aerosol-jet printing of graphene interconnects. Carbon N. Y. 91, 321-329 (2015).

19. Kosolia, C. T., EviVarka, M. \& Tsatsaroni, E. G. Effect of surfactants as dispersing agents on the properties of microemulsified inkjet inks for polyester fibers. J Surfact. Deterg. 14, 3-7 (2011).

20. Engberts, J. B. F. N. Applied surfactants principles and applications. By Tharwat F. Tadros. Angew. Chemie Int. Ed. 44, 5922-5922 (2005).

21. Panigrahi, P. K. Transport Phenomena in Microfluidic Systems. (Wiley, 2015). https://doi.org/10.1002/9781118298428.

22. Chang-jing, H. et al. Effect of the surfactants on atomization efficiency of lead zinc mineral powder suspension determined by atomic spectrometry. Rock Miner. Anal. 35, 245-250 (2016).

23. Lu, J. \& Corvalan, C. M. Coalescence of viscous drops with surfactants. Chem. Eng. Sci. 78, 9-13 (2012).

24. Oakley, C. \& Chahal, P. Aerosol jet printed quasi-optical terahertz components. IEEE Trans. Terahertz Sci. Technol. 8, 765-772 (2018).

25. Morales-Rodriguez, M. E., Joshi, P. C., Humphries, J. R., Fuhr, P. L. \& McIntyre, T. J. Fabrication of low cost surface acoustic wave sensors using direct printing by aerosol inkjet. IEEE Access 6, 20907-20915 (2018).

26. Hung, K. Y. et al. Investigation of ink modification for aerosol jet printing process on FR-4 substrate. Int. J. Adv. Manuf. Technol. 111, 1147-1156 (2020).

27. Rosker, E. S. et al. Approaching the practical conductivity limits of aerosol jet printed silver. ACS Appl. Mater. Interfaces 12, 29684-29691 (2020).

28. Krzemiński, J. Opracowanie technologii wysokoprzewodzących nanosrebrowych tuszów do wytwarzania elementów elektronicznych metodą druku aerozolowego. (Warsaw University of Technology, 2019).

29. Skarżyński, K. Effect of surfactant addition on aerosol jet printing ink properties. (Warsaw University of Technology, 2019).

30. Pietrikova, A. et al. Surface analysis of polymeric substrates used for inkjet printing technology. Circuit World 42, 9-16 (2016).

31. Fang, Y. et al. A novel, facile, layer-by-layer substrate surface modification for the fabrication of all-inkjet-printed flexible electronic devices on Kapton. J. Mater. Chem. C 4, 7052-7060 (2016).

32. Galstyan, V. et al. Well-ordered titania nanostructures for gas sensing. in Lecture Notes in Electrical Engineering vol. 268 LNEE 127-131 (Springer Verlag, 2014).

33. MacNeill, W., Choi, C. H., Chang, C. H. \& Malhotra, R. On the self-damping nature of densification in photonic sintering of nanoparticles. Sci. Rep. 5, 1-13 (2015).

34. Krzeminski, J. et al. Photonic curing of silver paths on 3D printed polymer substrate. Circuit World 45, 9-14 (2019).

35. Kasap, S. O. Principles of Electronic Materials and Devices. (McGraw-Hill, 2018).

36. Chen, G., Gu, Y., Tsang, H., Hines, D. R. \& Das, S. The effect of droplet sizes on overspray in aerosol-jet printing. Adv. Eng. Mater. 20, 1701084 (2018).

\section{Acknowledgements}

This research is part of the "Functional heterophase materials for structural electronics" project carried out within the First TEAM/2016-1/7 programme of the Foundation for Polish Science co-financed by the European Union under the European Regional Development Fund. The authors would like to thank the Institute of Micro and 
Sensor Systems, Otto-von-Guericke University, for big support in the conducting of the experiments featuring M3D Optomecs Aerosol Jet Printing system, and Helioenergia sp. z o.o., producer of Silvercon nAg, for providing silver nanopowder samples for the experiments.

\section{Author contributions}

K.S.-formal analysis, investigation, software, writing—original draft preparation, data visualisation. J.K.-conceptualisation, methodology, investigation, resources, validation, writing — review and editing. M.J.—conceptualisation, methodology. M.S._conceptualisation, validation, supervision, writing - review and editing, funding acquisition. All authors read and approved the final manuscript.

\section{Funding}

This research was funded by Foundation for Polish Science (Project No First TEAM/2016-1/7) and supported by the Institute of Metrology and Biomedical Engineering (Faculty of Mechatronics at the Warsaw University of Technology).

\section{Competing interests}

The authors declare no competing interests.

\section{Additional information}

Correspondence and requests for materials should be addressed to K.S. or M.S.

Reprints and permissions information is available at www.nature.com/reprints.

Publisher's note Springer Nature remains neutral with regard to jurisdictional claims in published maps and institutional affiliations.

Open Access This article is licensed under a Creative Commons Attribution 4.0 International ense, which permits use, sharing, adaptation, distribution and reproduction in any medium or format, as long as you give appropriate credit to the original author(s) and the source, provide a link to the Creative Commons licence, and indicate if changes were made. The images or other third party material in this article are included in the article's Creative Commons licence, unless indicated otherwise in a credit line to the material. If material is not included in the article's Creative Commons licence and your intended use is not permitted by statutory regulation or exceeds the permitted use, you will need to obtain permission directly from the copyright holder. To view a copy of this licence, visit http://creativecommons.org/licenses/by/4.0/.

(C) The Author(s) 2021 